\title{
Interface Modification in Type-II ZnCdSe/Zn(Cd)Te QDs for High Efficiency Intermediate Band Solar Cells
}

\author{
V.Deligiannakis $^{1,3}$, S. Dhomkar ${ }^{1}$, M. S. Claro ${ }^{1}$, I. L. Kuskovsky ${ }^{2,3}$, M. C. Tamargo ${ }^{1,3}$ \\ ${ }^{1}$ City College of CUNY, 160 Convent Ave., New York, New York 10031, USA \\ ${ }^{2}$ Queens College of CUNY, 65-30 Kissena Blvd, Queens, New York 11367, USA \\ ${ }^{3}$ The Graduate Center of CUNY, 365 Fifth Avenue, New York, New York 10016, USA \\ vdeligia@gmail.com
}

\begin{abstract}
A new growth process for type-II ZnCdSe/ZnCdTe quantum dots (QDs) is developed to avoid formation of deleterious straininducing ZnSe interfacial layer (IL) that forms during the migration enhanced epitaxy growth process used to form the QDs. This new growth sequence allows for improved control of the interfacial composition and simplifies the fabrication of the intermediate band solar cell device structure based on these QDs, since additional strain balancing schemes are no longer required to grow stress-free structures. In contrast to previous results, lattice-matched QD superlattices were obtained using near lattice-matched ZnCdSe barrier layers. X-ray diffraction and excitation intensity dependent photoluminescence studies were used to support such a conclusion. Our findings have applications for the growth of other heterointerfaces in which an undesirable IL may form due to lack of a common anion, and to desorption and incorporation of competing elements.
\end{abstract}

\section{Introduction:}

Intermediate band solar cells (IBSCs) have been proposed $^{1}$ as a possible solution to overcoming the Shockley-Queisser limit ${ }^{2}$ for solar cell quantum efficiencies. In these, a mid-gap energy band is formed, for instance by quantum dots within a relatively large bandgap semiconductor matrix. ${ }^{3} \quad$ Type-II ZnCdSe/ZnCdTe submonolayer QDs have been explored by our group for their promising properties as IBSCs. ${ }^{4,5,6}$ The $\mathrm{ZnCdSe}$ host material when lattice matched to InP has an energy bandgap of $\sim 2.1 \mathrm{eV}$ in which the ZnCdTe QDs can form an intermediate band with an energy $0.5-0.7 \mathrm{eV}$. The similarity of these parameters with those required for an ideal IBSC makes this material system an outstanding candidate. In the current state of IBSCs using type-I QDs grown by the Stranski-Krastanov (SK) method have shown significant advances. ${ }^{7,8}$ However, highly efficient devices have not you been realized. Due to the small size and low density of QDs, many layers are required to achieve an appreciable absorption response. The QDs grown by the SK method require the formation of a strained wetting layer, and so as the QD layers are repeated multiple times the strain increases, decreasing the overall quality of the material. ${ }^{9}$ Non-radiative processes are enhanced by defects that arise from strain within a layer. ${ }^{10}$ Furthermore, type-I band alignment systems that have been pursued are prone to exhibit Auger recombination, a non-radiative recombination process that can reduce the quantum efficiency of solar cell. This process is suppressed in typeII systems due to the separation of electrons and holes. ${ }^{11}$ By growing sub-monolayer type-II quantum dots by migration enhanced epitaxy (MEE) which proceeds via the Volmer Weber mechanism, one can avoid the formation of strained wetting layers thus minimizing strain effects, and also suppress Auger recombination phenomena.

However, other challenges arise during the growth of these materials due to the lack of a common anion as well as $\mathrm{Cd}$ desorption during the MEE growth of QDs. ${ }^{12,4}$ Typically, an unintentional $\mathrm{ZnSe}$ interfacial layer (IL) is formed at the interface of the $\mathrm{ZnCdSe}$ spacer material and the QDs, causing high tensile strain, modifying the band structure of the resulting materials. This requires adjustments in barrier and QD cadmium concentrations to fabricate stress free devices by strain balancing, ${ }^{6}$ complicating growth and increasing potential cost. The presence of the IL is also problematic due do its uncontrolled nature and its modification of the resulting band structure. Here we report the results of modified growth sequences that can suppress the formation of this $\mathrm{IL}$, allowing for better control of the QD band structure and eliminating the need for strain balancing schemes to obtain high structural quality.

\section{Growth:}

A series of $\mathrm{ZnCdSe} / \mathrm{ZnCdTe}$ QD superlattice (SL) samples were grown with varying source shutter sequences during the process of QD formation. A schematic of the structure is shown in Figure 1. The initial buffer layers and spacer material $(\mathrm{ZnCdSe})$ were grown by molecular-beam epitaxy (MBE), whereas to achieve the formation of sub- 

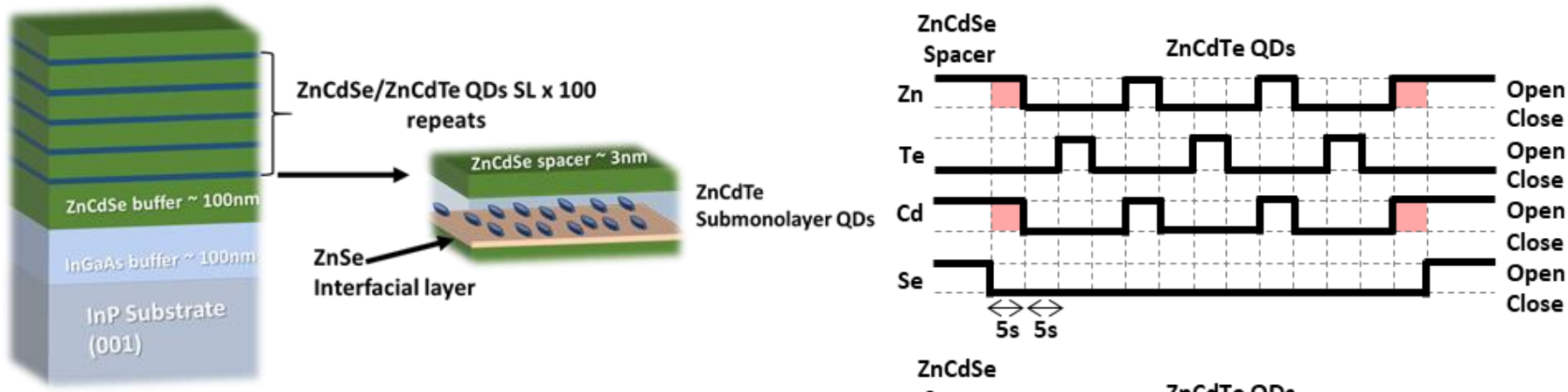

Figure 1 A schematic of type-II QD stacks, that would be used as an active region in an IBSC.

\begin{tabular}{cccccc}
\hline \hline & & Te Flux & & \\
Sample & $\mathrm{T}_{\mathrm{Te}}(\mathrm{C})$ & $\left(10^{-7}\right.$ Torr $)$ & Period & Cycle & Interface \\
\hline $\mathrm{A}$ & 270 & 9.0 & 50 & 4 & $\mathrm{Zn}, \mathrm{Cd}$ \\
$\mathrm{B}$ & 285 & 8.5 & 100 & 3 & $\mathrm{Cd}$ \\
$\mathrm{C}$ & 282 & 8.5 & 100 & 3 & $\mathrm{Cd}$ \\
$\mathrm{D}$ & 285 & 8.5 & 100 & 3 & $\mathrm{Cd}$ \\
$\mathrm{E}$ & 280 & 8.0 & 100 & 3 & $\mathrm{Zn}, \mathrm{Cd}$ \\
\hline \hline
\end{tabular}

Table I Relevant growth parameters for all samples.

monolayer ZnCdTe QDs the MEE process was implemented.

All samples were grown on (001) oriented InP substrates with a $100 \mathrm{~nm}$ lattice matched InGaAs buffer layer to ensure the formation of a high quality II-VI/III-V interface. The II-VI layer structure for all the samples includes a 100 $\mathrm{nm} \mathrm{ZnCdSe}$ buffer layer, followed by a superlattice consisting of alternating 2-4 $\mathrm{nm}$ thick $\mathrm{ZnCdSe}$ spacer layers and the submonolayer ZnCdTe QDs. The formation of $\mathrm{ZnCdTe}$ QDs was achieved by using a shutter sequence of alternating $\mathrm{Zn}, \mathrm{Cd}$ and Te fluxes with short wait times between them, with no impingent fluxes, to achieve MEE conditions.

The previously used shutter sequence developed by our group (shown in Fig. 2a) entailed a growth interruption with exposure of a $\mathrm{Zn}$ and $\mathrm{Cd}$ flux for $5 \mathrm{~s}$ after the growth of the $\mathrm{ZnCdSe}$ spacer, followed by a short 5 s wait time without any shutters open. ${ }^{6}$ Then the Te flux was opened for $5 \mathrm{~s}$ and followed again by a $5 \mathrm{~s}$ wait time without any impingent fluxes. This sequence was repeated several times (cycles). The number of cycles used were either three or four depending on the desired quantum dot size and composition. At the end of the MEE sequence the $\mathrm{Zn}$ and $\mathrm{Cd}$ shutters were opened simultaneously for $5 \mathrm{~s}$ before resuming the $\mathrm{ZnCdSe}$ spacer layer growth (by opening the

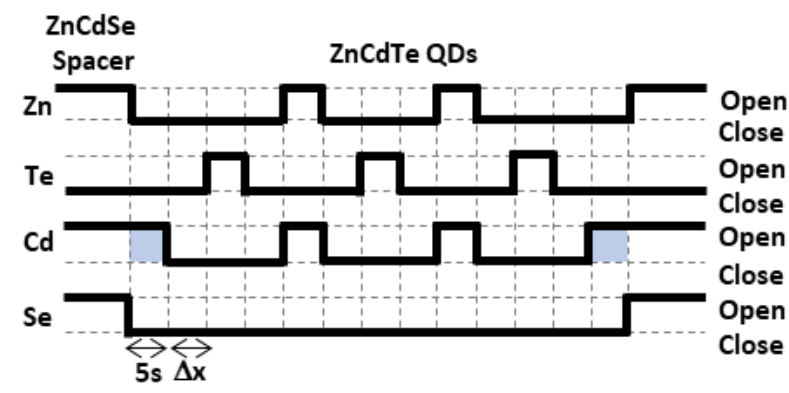

Figure 2 (a) Previously adopted growth shutter sequence (b) New shutter sequence used in the growth of sample $\mathrm{B}$ and $\mathrm{C}$, with $\mathrm{Cd}$ only shutter opened during the start and end of the MEE cycle; wait times of $\Delta x=$ 1 and $5 \mathrm{~s}$ were sued. Shaded boxes indicate open shutters for clarity.

Se shutter). The interruptions or "wait times" are intended, to allow excess $\mathrm{Zn}$ and $\mathrm{Cd}$ to desorb, and for the $\mathrm{Zn}, \mathrm{Cd}$ and Te to aggregate, and form quantum dots.

In this work, modifications of the shutter sequence (Fig 2b) using a 5s $C d$-only exposure between the growth of the spacer layer and the MEE cycles and reducing the "wait time" after the $\mathrm{Cd}$ exposure to $1 \mathrm{~s}$ were explored in an attempt to suppress the $\mathrm{ZnSe}$ tensile layer formation at the interface. Three samples (A, B and C) were grown for this comparison. Two additional samples, D and E, were also grown to obtain a lattice matched superlattice region using the unmodified growth sequence of sample A and the new optimum growth sequence of sample C. Table I summarizes some of the relevant growth parameters for the samples.

\section{Results and Discussion:}

Sample A was grown following the growth sequence of Figure 2a. Sample B was grown using the growth sequence shown in Figure 2b, with a Cd-only exposure and a $5 \mathrm{~s}$ "wait time" after the $\mathrm{Cd}$ exposure, and sample $\mathrm{C}$ was grown using the sequence of Figure $2 \mathrm{~b}$ but using only a $1 \mathrm{~s}$ "wait time" after the Cd-only exposure. HR-XRD 2 $\theta-\omega$ scans were taken around the (004) and (224) reflections to characterize the structural parameters of the grown samples. Figure 3 
shows the HR-XRD spectra for sampled A, B and C. Scans along the (004) symmetric reflection show the Bragg diffraction peaks of the various layers grown on InP substrates including the InGaAs and $\mathrm{ZnCdSe}$ buffer layers and the zeroth $\left(0^{\text {th }}\right)$-order superlattice peak $(\operatorname{SL}(0))$ as well as higher order satellite peaks, which originate from the periodic $\mathrm{ZnCdSe} / \mathrm{ZnCdTe}$ QD superlattice structure. Although the superlattice includes 2-4 nm ZnCdSe spacer it has been shown that as little as a single monolayer of $\mathrm{ZnSe}(\sim .3 \mathrm{~nm})$ can result in high tensile strain in contrast to the expected small compressive strain due to the $\mathrm{ZnCdTe}$ submonolayer QD material. This high tensile strain is evident by the appearance of the SL(0) peak at higher than the substrate Bragg angles, indicative of tensile strain.

The stress contribution from QDs is expected to be very small due to their submonolayer nature, so that the lattice constant of the SL is dominated by the IL and the composition of the spacer layer.

To estimate the strain accurately the (224) asymmetric reflection was also measured (Figure 3b). Using both (004) and (224) reflections one can calculate both the out-ofplane, $a_{\perp}$, obtained directly from the (004) reflection measurement, and the inplane, $a_{\|}$, lattice parameters. The latter is calculated as shown in Eq. 1 , where $d_{h k l}$ is the distance between crystallographic planes.

$$
\text { Eq. } \quad a_{\|}=\frac{8}{1 / d_{224}^{2}-1 / d_{004}^{2}}
$$

Then, one can calculate the "unstrained" lattice parameter, $a_{0}$, using Eq. 2 , where $C_{11}$ and $C_{12}$ are the elastic constants $C_{i j}$ of the layer of interest (SL(0) or $\mathrm{ZnCdSe}$ buffer) ${ }^{6}$

$$
E q .2 \quad a_{\|}=\frac{a_{\perp+\frac{2 C_{12}}{C_{11}} a_{\text {sub }}}}{1+\frac{2 C_{12}}{C_{11}}}
$$

Calculation of $a_{0}$ for the $\mathrm{Zn}_{\mathrm{x}} \mathrm{Cd}_{1-\mathrm{x}} \mathrm{Se}$ buffer is straight forward, since the elastic constants can be approximated by interpolation to the ternary composition. For the SL(0) peak, the elastic constants are estimated taking into account the $\mathrm{ZnCdSe}$ spacer layer, as well as the thickness of the interfacial layer present.

Comparing $a_{0}$ with the InP lattice constant we can see that the SL(0) peak for all the samples are nearly pseudomorphic with the InP substrate. The degree of relaxation of each of layer can be calculated by Eq.3,

$$
\text { Eq.3 R }=\frac{a_{\|}-a_{s u b}}{a_{0}-a_{s u b}}
$$

The results are shown in Table 2. As expected, the previously adopted growth sequence, used in sample A, did not suppress the formation of a $\mathrm{ZnSe}$ IL. In that sample, the higher order superlattice peaks are broad and weak, consistent with rough interfaces. The SL(0) peak for sample A shows a tensile strain of $2.00 \%$ with respect to the substrate. Using Vegard's law and assuming a fully strained IL we calculate that approximately 2 monolayers of $\mathrm{ZnSe}$ from at the interface between the spacer layer and QDs.
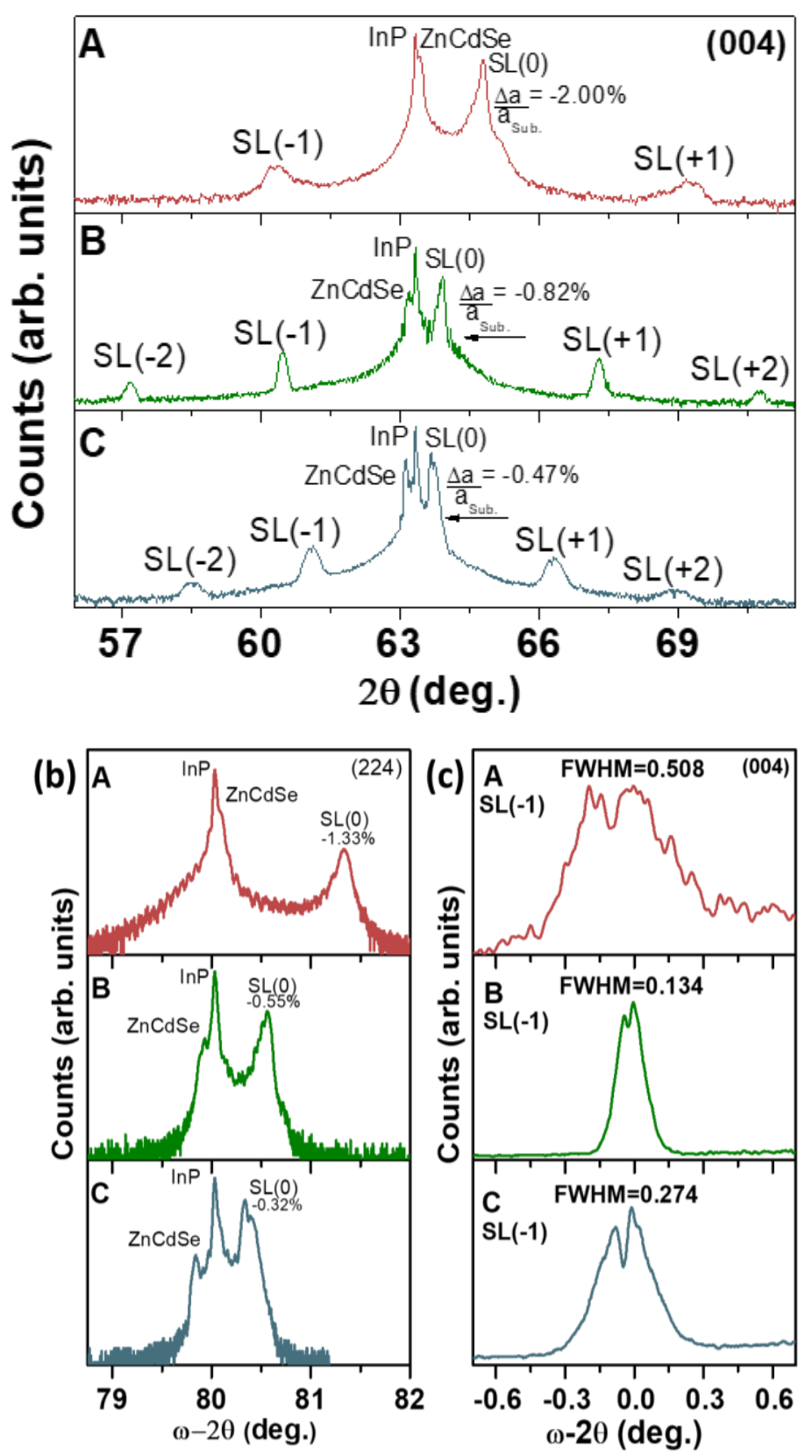

Figure 3 (a) HR-XRD along the (004) reflection; SL(0) mismatch is minimized with optimal growth sequence (sample C). (b) The (224) asymmetric reflection. (c) SL(-1) of the (004) reflection. 


\begin{tabular}{cccccc}
\hline \hline Sample & Layer & $\boldsymbol{a}_{\perp}$ & $\boldsymbol{a}_{\|}$ & $\boldsymbol{a}_{\mathbf{0}}$ & $\mathrm{R} \%$ \\
\hline \multirow{2}{*}{$\mathrm{A}$} & $\mathrm{ZnCdSe}$ & 5.865 & 5.865 & 5.865 & Matched \\
& $\mathrm{SL}(0)$ & 5.753 & 5.870 & 5.818 & Strained \\
& $\mathrm{ZnCdSe}$ & 5.880 & 5.864 & 5.871 & Matched \\
$\mathrm{B}$ & $\mathrm{SL}(0)$ & 5.820 & 5.871 & 5.849 & Strained \\
& $\mathrm{ZnCdSe}$ & 5.885 & 5.872 & 5.878 & $34 \%$ Relaxed \\
$\mathrm{C}$ & $\mathrm{SL}(0)$ & 5.840 & 5.872 & 5.858 & Strained \\
\hline \hline
\end{tabular}

Table II Lattice parameters and relaxation (R) for samples $\mathrm{A}, \mathrm{B}$ and $\mathrm{C}$.

By contrast, sample B shows a large reduction of the SL(0) mismatch as a result of having a Cd-only exposure during the initial growth interruption prior to the MEE cycles. Samples B and C show successively smaller tensile strains of $0.86 \%$ and $0.47 \%$, respectively, due to the suppression of the IL formation. The reduced strain results in a lower estimated $\mathrm{ZnSe}$ thickness of 1 and $\sim 0.75$ monolayers for samples B and C, respectively. We interpret this dramatic effect on the IL formation to the fact that $\mathrm{Zn}$ has a stronger affinity than $\mathrm{Cd}$ to bond with $\mathrm{Se}$ so it will displace $\mathrm{Cd}$ from $\mathrm{ZnCdSe}$ surface, and to the greater likelihood of $\mathrm{Cd}$ to desorb during the growth interruption when both the $\mathrm{Zn}$ and $\mathrm{Cd}$ shutters are open. ${ }^{13 \mathrm{~F} 12}$ When only the $\mathrm{Cd}$ shutter is open, as in sample $\mathrm{B}$, the competition between $\mathrm{Zn}$ and $\mathrm{Cd}$ is diminished and the effect of $\mathrm{Cd}$ desorption is suppressed. In sample $\mathrm{C}$, by also shortening the wait time between the $\mathrm{ZnCdSe}$ spacer and the start of the MEE cycle to $1 \mathrm{~s}$, instead of the $5 \mathrm{~s}$ used in sample $\mathrm{B}$, the desorption of $\mathrm{Cd}$ is further reduced. The satellite peaks of the superlattice SL(0) peak are narrower in samples B and $\mathrm{C}$, as is shown in figure $3 \mathrm{c}$, indicating smoother interfaces in those samples. From these results we conclude that the shutter sequence for sample $\mathrm{C}$ is optimal, since it results in the strongest suppression of the ZnSe IL.

It was previously reported that the high tensile strain produced by the IL could be strain compensated by growing the $\mathrm{ZnCdSe}$ spacer material with a large positive mismatch to InP. In order to achieve a lattice matched SL layer in the case of sample A it is necessary to use a strained $\mathrm{ZnCdSe}$ spacer layer composition that will strain balance the IL contribution. In figure 4, we show a comparison of the XRD scans for samples D and E, which were designed to obtain lattice matched $\operatorname{SL}(0)$ peaks, and were grown using the

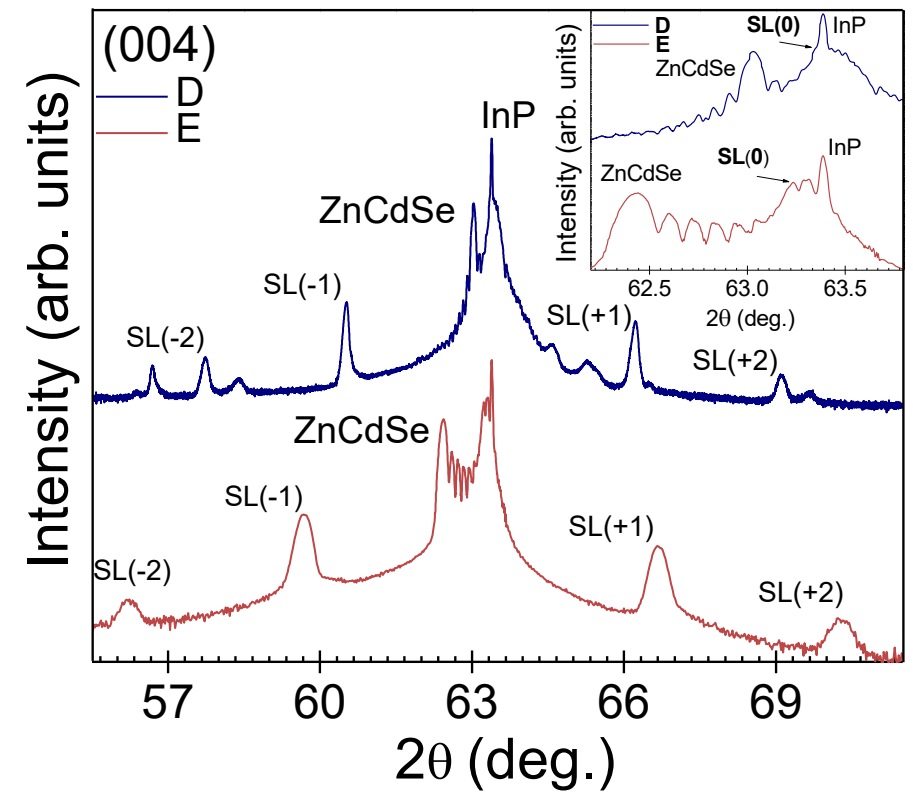

Figure 4 HR-XRD for sampled D and E along the 004 reflection. The inset focuses on the near lattice matched SL(0) peak for the two samples.

growth sequences of samples $\mathrm{C}$ and $\mathrm{A}$, respectively. For sample $\mathrm{E}$ the spacer layer was grown with a $\mathrm{ZnCdSe}$ composition such that it has a lattice mismatch of $+1.35 \%$. As a result, the SL(0) peak is closely lattice matched to InP due to strain compensation of the $\mathrm{ZnSe}$ IL by the compressively strained spacers. In contrast, in sample D, which was grown using the new optimum growth sequence of sample $\mathrm{C}$ described above, the lattice mismatch for the $\mathrm{ZnCdSe}$ spacer layer needed to obtain a lattice matched superlattice layer is only $\sim 0.5 \%$. The improved material quality of sample $\mathrm{D}$ is evident from the sharp superlattice peaks observed by $\mathrm{XRD}^{14}$ (Figure 4). Thus, the material resulting from the new modified shutter sequence has a greatly reduced unintentional $\mathrm{ZnSe}$ IL, requiring minimal strain compensation, and leading to an improved overall structural quality.

To verify that the new shutter does not affect the type-II band alignment and retains the favorable optical properties needed for the IBSC we measured the PL spectra for sample D. Figure 5 shows the $77 \mathrm{~K}$ excitation intensity dependent PL emission which was taken by using a $50 \mathrm{~mW} 405 \mathrm{~nm}$ diode laser and collected with a Ocean Optics 2000 spectraomter. A strong broad PL emission typical of typeII structures is seen ${ }^{15,16}$. The $\mathrm{ZnCdTe}$ QD PL is centered around $1.92 \mathrm{eV}$. A small peak at $2.14 \mathrm{eV}$ can be observed at high excitations, which we attribute to the $\mathrm{ZnCdSe}$ spacer and /or buffer layer. Furthermore, as expected for type-II hetero structures, the intensity dependent blue shift of the PL emission peak is clearly observed (Fig. 5 b). Our results 

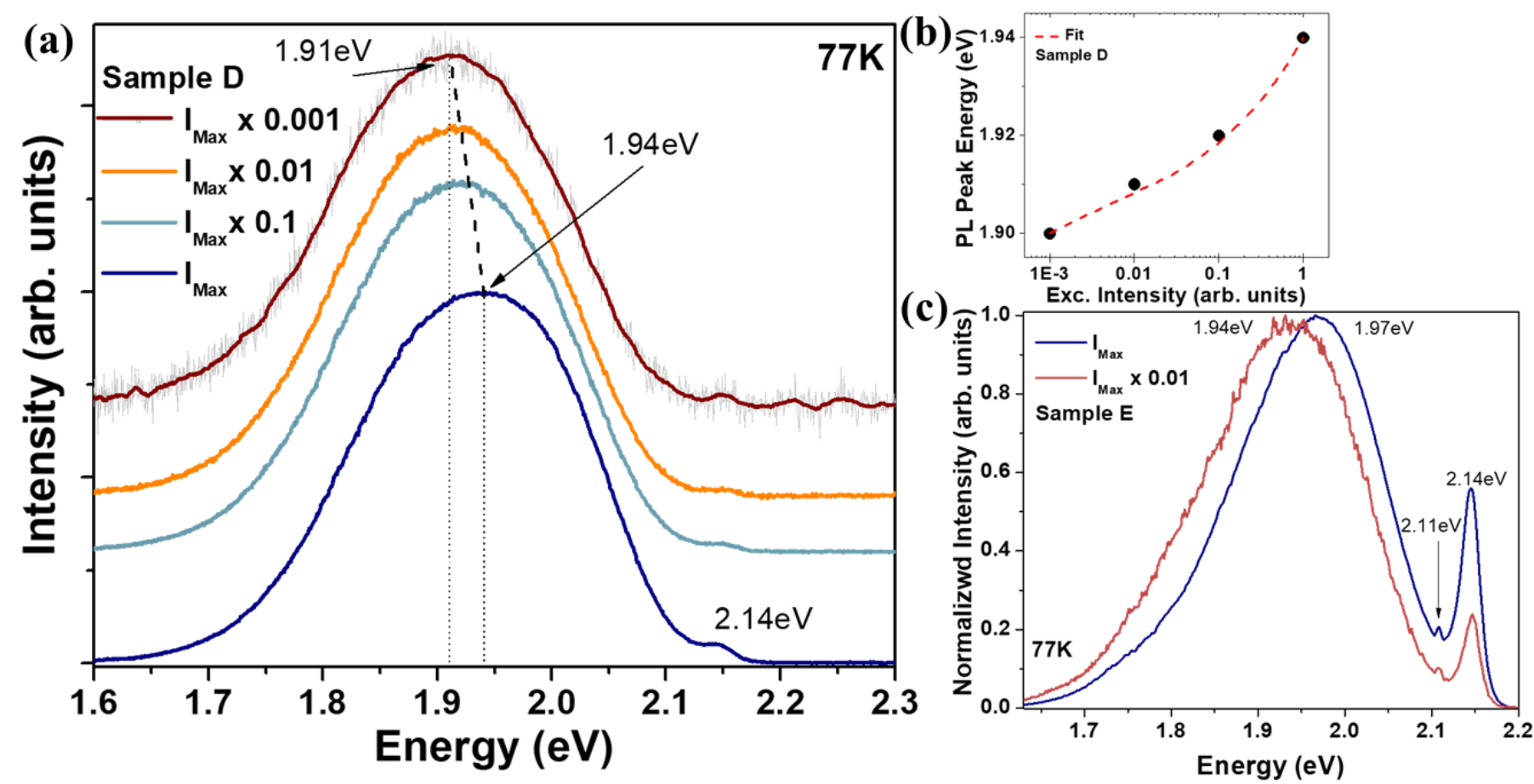

Figure 5 (a) Low temperature intensity dependent PL for sample D confirms the type-II band alignment between QDs and host material. (b) (c)

indicate that the materials grown with the modified shutter sequence, which have improved structural properties, with greatly reduced formation of the deleterious $\mathrm{ZnSe} \mathrm{IL}$ retain the good optical properties typical of these type-II QD systems. Sample E, which was the Thus, we conclude that this new growth sequence is favorable for the design of an optimum IBSC.

\section{Conclusion:}

We have modified the MEE shutter sequence used in the growth of submonolayer ZnCdTe type-II QDs for use in IBSCs in order to reduce the formation of a deleterious $\mathrm{ZnSe}$ interfacial layer that was previously reported. We have shown that a Cd-only exposure at the start of the MEE shutter sequence, along with a reduced "wait time" after the $\mathrm{Cd}$ exposure greatly suppresses the $\mathrm{ZnSe}$ IL formation. The results are consistent with the suppression of $\mathrm{Cd}$ depletion from the $\mathrm{ZnCdSe}$ spacer region at the start of the growth interruption prior to the MEE growth sequence. We propose that two processes are responsible for the $\mathrm{Cd}$ depletion during the $\mathrm{Zn}$ and $\mathrm{Cd}$ exposure of the previously used growth sequence: $\mathrm{Cd}$ desorption due to its small sticking coefficient at the growth temperatures, and replacement of $\mathrm{Cd}$ by the $\mathrm{Zn}$ in the impingent flux, due to the stronger affinity of $\mathrm{Zn}$ than $\mathrm{Cd}$ to bond to Se. The resultant materials have improved structural properties while maintaining their good optical characteristics. The results presented here have implications in other heterostructure systems in which the lack of a common anion results in unwanted interfacial layer formation. 


\section{References:}

1 A. Luque, and A. Marti, Phys. Rev. Lett., 78, 5014 - 5017 (1997).

2 W. Shockley, and H.J. Queisser, J. Appl. Phys. 32, no. 3: 510 (1961).

3 A. Luque, A. Martí, and C. Stanley, Nature Photonics, 6(3), 146-152 (2012).

4 S. Dhomkar, U. Manna, L. Peng, R. Moug, I. C. Noyan, M. C. Tamargo, and I. L. Kuskovsky, Solar Energy Materials Solar Cells, 117, 604-609 (2013)

5 S. Dhomkar, I. L. Kuskovsky, U. Manna, I. C. Noyan, and M. C. Tamargo, Journal of Vacuum Science \& Technology B, Nano. and Micro.: Mat., Process., Meas., and Phen., 31(3), 03C119(2013).

6 C. M. Imperato, G. A. Ranepura, L. I. Deych, and I. L. Kuskovsky, Jou. Elec. Mat., 47, 4325-4331 (2018).

7 Z. Zheng, H. Ji, P. Yu, and Z. Wang, Nano. Res. Lett.11(1) (2016).

8 Y. Okada, N. J. Ekins-Daukes, T. Kita, R. Tamaki, M. Yoshida, A. Pusch, O. Hess, C. C. Phillips, D. J. Farrell, K. Yoshida, N. Ahsan, Y. Shoji, T. Sogabe, and J. F. Guillemoles, App. Phys. Rev. 2, 021302 (2015).

9 T. Sugaya, O. Numakami, S. Furue, H. Komaki, T. Aman, K. Komori and S. Niki, 37th IEEE Photo. Spec. Conf. (2011).

10 A. Marti, N. López, E. Antolín, E. Cánovas, A. Luque, C. R. Stanley, C. D. Farmer, and P. Díaz, Appl. Phys. Lett. 90, 233510 (2007).

11 G. G. Zegrya, \& A. D. Andreev, App. Phys. Lett., 67(18), 2681-2683 (1995).

12 B. Bonef, B. Haas, J.-L. Rouviere, R. Andre, Bougerol, C., Grenier, A. Zuo, J.-M. Journal of Microscopy, 262(2), (2016).
13 E. M. Larramendi, O. de Melo, and I. HernándezCalderón, Phys. Stat. Sol. (b), 242(9), 1946-1950(2005).

14 S. M. Hubbard, C. G. Bailey, C. D. Cress, S. Polly, J. Clark, D. V. Forbes, Wilt, D. M. 33rd IEEE Photovolatic Specialists Conference (2008).

15 F. Hatami, M. Grundmann, N. N. Ledentsov, F. Heinrichsdorff, R. Heitz, J. Böhrer, Z. I. Alferov Physical Review B, 57(8), 4635-4641, (1998)..

16 S. Dhomkar, H. Ji, B. Roy, V. Deligiannakis, A. Wang, M. C. Tamargo, I. L. Kuskovsky. Journal of Crystal Growth, 422, 8-14, (2015). 